\title{
Effect of Progressive Muscle Relaxation on DASS Score and Reaction Time
}

\author{
Anitha O.R. ${ }^{1}$, Umadevi B. ${ }^{1}$, Christofer Thomas ${ }^{2}$ \\ ${ }^{1}$ Associate Professor, ${ }^{2}$ Assistant. Professor, Dept. of Physiology, \\ Sapthagiri Institute of Medical Sciences \& Research Centre
}

\begin{abstract}
Background: Reaction time is a reliable indicator of rate of processing of sensory stimuli by central nervous system and its execution in the form of motor response. It is found to be affected by factors like physiological stress, mood and diseases like neuropathy \& nerve injury. Various relaxation interventions have shown to reduce stress, but there are fewer reports about the effect of progressive muscle relaxation on stress and reaction time.
\end{abstract}

Objective: The present investigation was undertaken to study the effect of progressive muscle relaxation on visual and auditory reaction times (RTs) \& on Depression Anxiety Stress Scale (DASS) Score.

Method: 66 students who had moderate to severe stress \& who met the inclusion \& exclusion criteria were enrolled in the study and were randomly divided into 2 groups- subjects \& controls. 33 subjects were given progressive muscle relaxation training daily for 6 weeks. DASS score, visual \& auditory reaction time were recorded before $\&$ after the training period. The readings were compared within the group $\&$ also with the age matched 33 controls who did not receive Progressive muscle relaxation training.

Results \& Interpretation: In the study group, there was decrease in the DASS score after the training. There was a significant decrease $(\mathrm{P}<0.001)$ in visual RT (from $255.4 \pm 58.0 \mathrm{~ms}$ to $208.6 \pm 37.8 \mathrm{~ms}$ ) as well as auditory RT (from $225.2 \pm 061.4 \mathrm{~ms}$ to $187.9 \pm 038.5 \mathrm{~ms}$ ). whereas the change in reaction time \& DASS score was not significant in the control group.

Conclusion: Decrease in DASS Score \& reduction in reaction times following progressive muscle relaxation technique, shows that it is a very effective tool in decreasing the stress levels in individuals. Progressive muscle relaxation is an easy and inexpensive method as it may help students to concentrate better \& excel in their studies.

Keywords: Stress, Reaction time, Progressive muscle relaxation, DASS Score.

\section{Introduction}

Stress has become an inevitable component of one's life. The term "stress", coined by Hans Syle is defined as "the non-specific response of the body to any demand for

\section{Corresponding Author: \\ Dr. Anitha O.R.}

Associate Professor, Dept. of Physiology, Sapthagiri Institute of Medical Sciences \& Research Centre, Bangalore change." Gratuitous levels of stress results in detrimental consequences on the quality of life of an individual. These negative effects are manifested in physical health, learning ability and retention, interpersonal relationships, and behavior. ${ }^{1}$ Some individuals even indulge in self-destructive coping mechanisms such as alcohol consumption or smoking in order to alleviate these effects of stress and anxiety. In order to prevent these unhealthy behaviors, various relaxation techniques have been used by the researchers and it has been shown that these techniques help the individuals in positive coping and also been shown to reduce stress. 
Method for relaxation can range from various breathing patterns to pleasant visual imagery exercises. These method are also widely used to treat a variety of health problems including headaches, anxiety, and insomnia. Not only do relaxation techniques reduce bad habits and treat some common health problems, but they have also been proven to be effective for relieving stress and anxiety. ${ }^{1}$

Reaction time(RT) is a reliable indicator of rate of processing of sensory stimuli by central nervous system and its execution in the form of motor response. Auditory $\&$ visual reaction times are the frequently used RTs; shorter RT means better performance which is indeed a prerequisite for surgeons \& other professionals. It is altered by number of factors; stress being one among them. Psychological distress such as stress, anxiety and depression are frequent among medical students the reason being peer pressure and academic burden which can affect their academic performance, physical health and psychosocial wellbeing. ${ }^{2} 1^{\text {st }}$ year medical students are exposed to huge syllabus in a shorter span of time, that induce stress in them. Though stress in minimal level is facilitatory to the brain, increased stress can be detrimental; it can manifest as Anxiety/Depression disorders. Increased stress increases reaction time \& decreases the attention span $\&$ concentration. ${ }^{2}$

There is a need for intervention that can not only decrease stress, but also improve reaction time. In this measure, lot of interventions has been tried in the form of meditation, music, yoga, etc. Progressive muscle relaxation developed by Edmund Jacobson, involves tensing \& relaxing the muscles, one group of muscle/one body part at a time, to bring about a feeling of physical relaxation. ${ }^{3}$ Different relaxation techniques have shown to reduce stress, but less has been proven about the effect of this less known technique of progressive muscle relaxation on stress and reaction time.

Hence this study was designed to explore the effect of progressive muscle relaxation technique on psychological stress and reaction time in $1^{\text {st }}$ year Medical students.

Subjective assessment of stress was done by self rated DASS Score. It is a reliable indicator of the level of stress and is also easy to administer. DASS Questionnaire is a set of three self report scales designed to measure the negative emotional states of depression, anxiety and stress with 7 items per scale. ${ }^{4}$ Reaction time measurement was used as objective assessment method to see the effect of Relaxation technique.

\section{Materials and Method}

The present study was undertaken in a medical college in south India. The study was approved by the institutional ethics Committee. The informed written consent was taken from all the subjects who volunteered to participate in the study. 150 students were given the DASS questionnaire \& proforma to fill. 130 students returned the filled forms.

Detailed medical history of subjects was taken, which included history of present illness, past history, personal history (diet, sleep pattern, exercise \& sports habits, vehicle driving, addiction). They were further recruited based on the following criteria:

Inclusion Criteria: Students who had moderate to severe stress scores that is between 19-33 scoring according to DASS score for stress.

Subjects aged between $17-21$ yrs who gave informed consent.

Exclusion Criteria: Any medical or surgical disease (hypertension, diabetes, tuberculosis, psychiatric disorders, head injury, epilepsy, drug therapy etc.) E.N.T. diseases and ophthalmic diseases which would affect reaction time of the individual.

The following protocol was used. 


\section{Study Protocol:}

\section{0 students Recruited}

DASS Score

Moderate to severe stress

$\mathrm{N}=66$, Random sampling

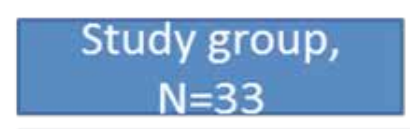

Progressive

muscle relaxation
Control group,

$\mathrm{N}=33$

No intervention
Mild stress, $\mathrm{N}=64$

\section{DASS Score and Reaction time}

Reaction time recording: The reaction time was recorded in a calm cool place between 11.00 am -12.30 pm hours of the day. Reaction Timer: The device used in the present study to measure Auditory and visual reaction time is a $\mathrm{PC} 1000$ reaction timer. $\mathrm{PC} 1000$ is a 1000 hertz square wave oscillator which has a soft key for 'start 'and 'stop' function. This instrument has two components (A \& B) .Component (A) has a start button which is handled by the examiner only, second component (B) has a stop button which is handled by the subject. Small red LED light was used as visual stimulus. A $1000 \mathrm{~Hz}$ tone through the head phone was the auditory stimulus. Audacity software was used which records the reaction time in $0.001 \mathrm{sec}$ accuracy in wave format Visual Reaction Time (VRT)Measurement: The Examiner pressed the 'start' button in the component (A) \& the subject was instructed to press the 'Stop' button in component (B) with the right index finger first as soon as he/she sees the red light in the instrument. Visual Reaction time was recorded in audacity software.

Auditory Reaction Time (ART) Measurement: The Examiner pressed the 'start' button and the subject was instructed to press the stop button with the right index finger first as soon as he hears the sound (1000 hertz's tone) through the head phone connected to it. Auditory Reaction time was recorded in Audacity software.

Five trials were given for measurement of VRT \&
ART. The mean of the five reaction times was taken as the final reaction time.

Progressive muscle relaxation technique: Progressive muscle relaxation uses tension and release of muscles throughout the body to relax. It teaches how to relax the muscles through a two step process. ${ }^{3}$ First, we systematically tense particular muscle groups in the body, next, we release the tension and notice how muscles feel when they are relaxed. These practice sessions were done using the video demonstration. The students were made to practice the muscle relaxation technique at the same time daily for 6 weeks under the guidance of researchers.

Students were given the handouts of the guidelines to perform the relaxation technique and also the video clipping, so as to facilitate them to continue their relaxation techniques at home/hostel in case of holidays or their absence.

\section{Results}

66 students were randomly divided into two groups as cases \& controls with 33 members in each. Cases were those who practiced the progressive muscle relaxation technique for 6 weeks. Controls were those who did not practice relaxation.

Earlier to intervention the reaction time was not significantly different among both the groups. (Table 1). 
Table 1: Pre intervention reaction time in cases \& controls

\begin{tabular}{|l|c|c|c|}
\hline Reaction time (in Milliseconds) & Cases & Controls & p value \\
\hline Visual & $255.4 \pm 58.0$ & $255.0 \pm 60.5$ & 0.98 \\
\hline Auditory & $225.2 \pm 61.4$ & $231.2 \pm 48.2$ & 0.66 \\
\hline
\end{tabular}

$\mathrm{p}$ Value $<0.05$ is statistically significant

Table 2: Reaction time in Controls

\begin{tabular}{|c|c|c|c|c|}
\hline Reaction time (in Milliseconds) & $\mathbf{N}$ & Mean & Std. Deviation & p value \\
\hline Visual Reaction time pretest & 33 & 255.0 & 60.515 & \multirow{2}{*}{0.13} \\
\hline Visual Reaction time posttest & 33 & 260.0 & 57.371 & \\
\hline Auditory Reaction time Pretest & 33 & 231.2 & 48.293 & \multirow{2}{*}{0.86} \\
\hline Auditory Reaction time Posttest & 33 & 232.1 & 47.125 & \\
\hline
\end{tabular}

Table 3: Reaction time in Cases before(Pre) $\&$ after(Post) the intervention

\begin{tabular}{|l|l|l|l|l|}
\hline Reaction time (in Milliseconds) & N & Mean & Std. Deviation & P value \\
\hline Visual Reaction time pretest & 33 & 255.4 & 58.478 & \multirow{2}{*}{0.01} \\
\cline { 1 - 2 } & 33 & 208.7 & 37.828 & \multirow{2}{*}{0.01} \\
\cline { 1 - 3 } & 33 & 225.3 & 61.423 & \\
\cline { 1 - 3 } & 33 & 187.9 & 38.543 & \\
\hline
\end{tabular}

From the above tables $1,2 \& 3$ it is clear that the the reaction times decreased significantly in the study baseline reaction times were not significantly different group who practiced progressive muscle relaxation as between the control and study group. After 6 weeks, compared to controls.

Effect of Progressive muscle relaxation on Reaction times:

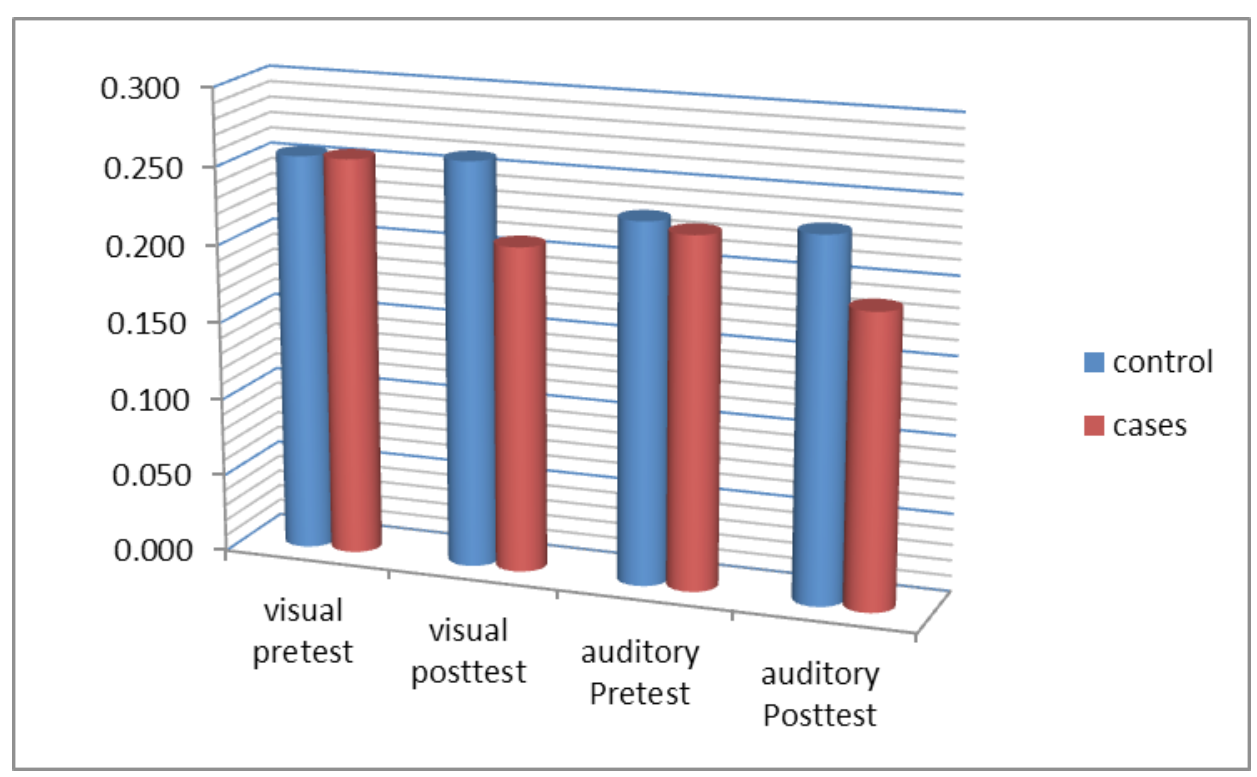


Effect of progressive muscle relaxation on DASS Score:

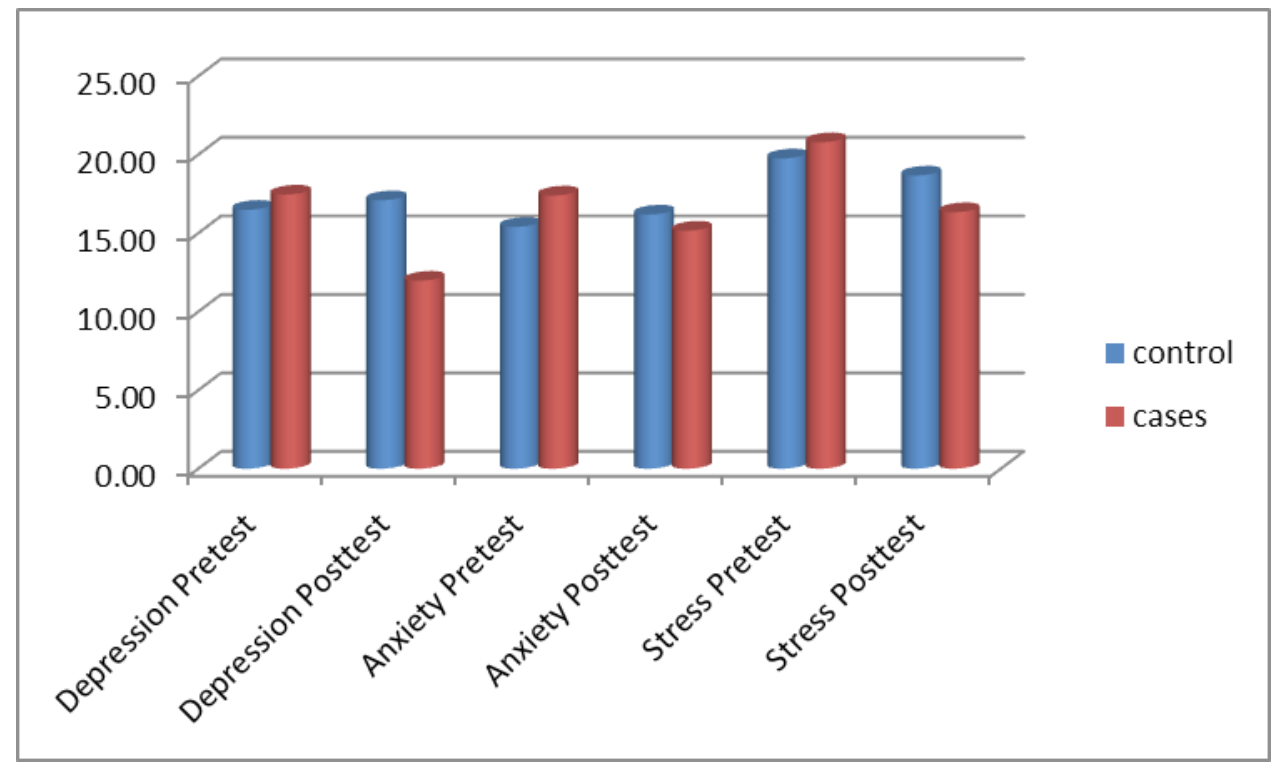

Table 4: DASS Score after progressive muscle relaxation in cases in comparision with controls

\begin{tabular}{|l|c|c|}
\hline Mean Scores & Control & Cases \\
\hline Depression Pretest & 16.48 & 17.45 \\
\hline Depression Posttest & 17.09 & 11.97 \\
\hline Anxiety Pretest & 15.39 & 17.39 \\
\hline Anxiety Posttest & 16.18 & 15.15 \\
\hline Stress Pretest & 19.76 & 20.79 \\
\hline Stress Posttest & 18.67 & 16.33 \\
\hline
\end{tabular}

There was decrease in all the three scores; depression, anxiety \& stress scores after PMR. The decrease in depression scores was much higher, followed by stress and anxiety scores.

\section{Discussion}

In the present study Pre intervention scores of reaction times and the DASS score between controls \& cases were not statistically different. Post intervention, there was significant decrease in depression, anxiety \& stress score in the study group. Similar findings were recorded in the study done by Praseeda P. et. al. wherein they found the post test mean score of academic stress was significantly lower than pre-test score which clearly proved that Progressive Muscle Relaxation was effective in reducing academic stress in classroom situation. ${ }^{6}$

Similar decrease in stress due to music was noted by Stephene Khalfa \& others, where relaxing music decreased the Cortisol level. ${ }^{7}$
Stress is a word derived from the Latin word 'stringere', meaning to draw tight. Progressive muscle relaxation (PMR) technique is based on the premise that muscle tension is the body's psychological response to anxiety-provoking thoughts and that muscle relaxation blocks anxiety. This technique involves learning to monitor the tension in specific muscle groups by first tensing each muscle group. This tension is then released, as attention is directed towards the differences felt during tension and relaxation. PMR brings about a feeling of physical relaxation. When the body is physically relaxed, anxiety is also reduced. ${ }^{8}$

Researchers have found that medical science students have higher levels of anxiety \& stress than other university students. ${ }^{9}$ This could have negative effects on the student, their family members and their classmates. Thus practicing PMR on regular basis could bring down the stress \& anxiety in medical students.

Studies have attempted to make correlations with 
physiological and behavioral responses to stress and relaxation in order to improve performance abilities. A reduction in reaction time was seen with studies that reduced environmental stress. ${ }^{10}$

In our study, Post intervention, along with decrease in stress, there was significant decrease in both reaction times: visual $\&$ auditory. A decrease in reaction time indicates an improved sensory motor performance and could be due to an enhanced processing ability of the central nervous system. This effect on central nervous system could be due to greater and improved concentration power and inhibition of extraneous stimuli indicating better attention and less distractibility.

Similar findings were found by Borkar and Pednekar who studied the changes on Visual reaction time (VRT) and auditory reaction time (ART) before and after 4 weeks of pranayamic breathing exercises. ${ }^{11}$

Similar reduction in reaction times were also observed by Begum N.,et.a l. after short session of yoga, wherein the authors attributed it to the stimulation of the vagal nerves, restoring activities of Parasympathetic Nervous System (PNS) and the Gamma Amino-Butyric Acid (GABA) systems. Added to this, there is increase in the cerebral blood flow particularly in right hemisphere frontal lobe which may improve reaction time. ${ }^{12}$

Another study on yoga-based relaxation techniques in relation to attention speed and information processing showed a significant increase in scores on a six-letter cancellation task as competition. In this study by P. Subramanya and et. al., the supine rest group showed an increase in performance on tasks involving strict motor speed but not on a digit substitution task, which relies more heavily on cognitive function. This suggests differing effects of relaxation based on task type. ${ }^{13}$

Malathi and Parulkar demonstrated a significant decrease in reaction times both after single session of yogasana $\&$ also after 6 weeks of training. ${ }^{14}$

The medical students get trained to reduce their patient's stress and anxiety and thus improve patient's quality of life. In the meantime the health of medical students and their anxieties are being ignored. Practicing Progressive muscle relaxation technique can help the medical students not only in combating this anxiety and stress but also improve the reaction time, helping them in better judgment and quick action.
Strength of the study: The main advantage of using Progressive muscle relaxation technique which we observed was better compliance of students towards the practice of this procedure. The students could follow the steps well as it was a guided relaxation video. They neither felt sleepy nor distracted as they were actively involved in the contraction \& relaxation of group of muscles as contrary to other techniques which use plain music.

\section{Conclusion}

Our results show that practicing progressive muscle relaxation results in significant reduction in visual and auditory RTs and also the DASS Score. Thus we can conclude progressive muscle relaxation is a very effective tool in decreasing the stress levels in individuals. It will not only help students to concentrate better \& excel in their studies but also is useful for sportsmen and surgeons.

Limitations of this study: The present study is a case-control study where the subjects were randomly selected from the population. The sample size is very small. The study can be extended to a larger population, so that results could be generalized.

Acknowledgements: The authors thankfully acknowledge subjects for volunteering to participate in the study and also Professor, Dr. Mary Prem Jayarajan for her continuous support and Mrs. Lavanya for statistical analysis.

\section{Conflict of Interest: None}

\section{Sources of Funds: Nil}

Ethical Clearance: Institutional Ethical Committee, SIMS \& RC, Bangalore.

\section{References}

1. Jacobs GD, Friedman R . EEG spectral analysis of relaxation techniques. Appl Psychophysiiol Biofeedback. 2004;29: 245-254.

2. Nahi Salam A, Yousuf R, Bakar SMA, et.al.. Stress among Medical Students in Malaysia: A Systematic Review of Literatures. Int.Med. J Malays.. 2013; 20 (6): $649-55$.

3. Pagnini, F., Gian, M.M., Castelnuovo, G., \& Molinari, E. (2009). Efficacy of relaxation training in treating anxiety. International Journal 
of Behavioural Consultation and Therapy, 5(3-4), 264-269.

4. Lovibond, S.H. \& Lovibond, P.F. Manual for the Depression Anxiety \& Stress Scales.1955. (2nd Ed.)Sydney: Psychology Foundation.

5. Niruba R, Maruthy KN. Assessment of Auditory and Visual Reaction time in Type 2 diabetics Al Ameen J Med Sci 2011;4(3):274-79.

6. Nair PP, Meera KP. Effectiveness of Progressive Muscle Relaxation in Reducing Academic Stress of Secondary Schools.,IOSR-JHSS.2014; 19(8) : 2932 .

7. Khalfa S, Dalla S.Effect of relaxing music on salivary Cortisol level after relaxing music. Ann. N.Y. Acad. of Sci.2003: 374-376

8. Rausch, S. M., Gramling, S. E., \& Auerbach, S. M. Effects of a single session of large-group meditation and progressive muscle relaxation training on stress reduction, reactivity, and recovery. Int. J. Stress Manag.. 2006.13(3), 273-290.
9. Nayeri ND, Hajbaghery MA.Effects of progressive relaxation on anxiety and quality of life in female students: A non-randomized controlled trial. Complement. Ther. Med. 2011 19: 194-200

10. Eason RL, Brandon JE, Smith TL, et.al. Relaxation training effects on reaction/response time, frontalis EMG, and behavioral measures of relaxation with hyperactive males. Adapt. Phys. Act. Q.1986; 3: 329-341.

11. Borker A Pednekar J. Effect of pranayama on visual and auditory reaction time. IJPP.2003; 47:229-30.

12. Begum LN,Kumaran DS, Venkatesh G, et al., Short Course of Yoga to Improve Reaction Time JCDR, 2012 September; 6(7): 1241-1243

13. Subramanya $P \&$ Telles $S$ Effect of two yoga-based relaxation techniques on memory scores and state anxiety. Bio Psycho Social medicine. 2009; 59:3-8.

14. Malathi, Parulkar G V, Effect Of Yogasanas On The Visual And Auditory Reaction Time. IJPP, 1989; 33 (1) 110-112. 